\title{
On the nature of the Herbig Be star V 380 Orionis $^{\star, \star \star}$
}

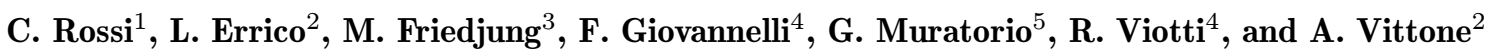 \\ 1 Istituto Astronomico, Università La Sapienza, Via G.M. Lancisi 29, 00161 Roma, Italy \\ 2 Osservatorio Astronomico di Capodimonte, Via Moiariello 16, 80131 Napoli, Italia \\ 3 Institut d'Astrophysique, CNRS, 98bis Boulevard Arago, 75014 Paris, France \\ 4 Istituto di Astrofisica Spaziale, CNR, Area di Ricerca Tor Vergata, Via del Fosso del Cavaliere, 00133 Roma, Italy \\ 5 Observatoire de Marseille, 2 place Le Verrier, 13248 Marseille Cedex 04, France
}

Received August 11, 1997; accepted December 16, 1998

\begin{abstract}
We discuss new and archive spectroscopic and photometric observations of the Herbig Ae star V 380 Ori obtained between 1978 and 1995 and covering at different epochs the wavelength range from $1200 \AA$ to $5 \mu \mathrm{m}$. The coordinated JHKLM and CVF infrared observations and optical spectroscopy made at ESO of March 1985 confirm the presence of a strong IR excess due to emission from hot cicumstellar dust. The comparison with IR photometry from the literature suggests the presence of oscillations, without secular variations. The optical spectrum of V 380 Ori, observed at ESO during 1983-1985, and at OHP in January 1995, remained substatially stable, and was all the time characterized by a large number of broad $\left(F W H M \sim 150 \mathrm{~km} \mathrm{~s}^{-1}\right)$ permitted emission lines, probably formed in a rotating optically thick disk. The strongest Fe II lines have complex profile with a principal central emission and a blue-shifted $\left(-140 \mathrm{~km} \mathrm{~s}^{-1}\right)$ wind component, the redward component probably being occulted by the disk. The multiplet 42 lines have P Cygni absorption components shifted by $-250 \mathrm{~km} \mathrm{~s}^{-1}$ which should be associated with a teneous cool wind. We remark the secular behaviour of the stellar activity probe He I $5876 \AA$ line, which is present in all our spectra as a broad emission, while in other times it was absent or in absorption. The ultraviolet (IUE) spectrum shows a short wavelength cut off at $\sim 1300 \AA$ typical of a B9-A0 star, with a rich absorption spectrum, which is thought to be produced in an optically thick stellar envelope or wind. The $2175 \AA$ interstellar band strength is consistent with a mean
\end{abstract}

Send offprint requests to: C. Rossi, rossic@uniroma1.it

* Based on observations made with the International Ultraviolet Explorer collected at the Villafranca Satellite Tracking Station of ESA, on archive IUE data, and on observations collected at the European Southern Observatory, at the Observatoire de Haute Provence.

$\star \star$ Table 3 is only available in electronic form at the CDS via anonymous ftp to cdsarc.u-strasbg.fr (130.79.128.5) or via http://cdsweb.u-strasbg.fr/Abstract.html galactic-type extinction law with an $E_{B-V}=0.20 \pm 0.05$, much smaller than that derived from the visual $(\sim 0.5)$, which suggests an anomalous local UV extinction. The comparison of the archive IUE data shows that during 1978-1989 there was no significant flux variation, with the possible exception of the UV Mg II emission line.

Key words: stars: emission-line, Be - stars: individual: V 380 Ori — stars: pre-main sequence — infrared: stars - Ultraviolet: stars

\section{Introduction}

V 380 Ori belongs to the class of the Herbig Ae/Be stars, defined by Herbig (1960). Such stars have an early spectral type with emission lines, lie in an obscured region and illuminate fairly bright nebulosity in their immediate vicinity. They generally lie above the zero age main sequence in the Hertsprung-Russel diagram and are thought to be in an early pre-main sequence evolutionary stage (e.g. Finkenzeller \& Mundt 1984). According to Böhm \& Catala (1995) Herbig Ae/Be stars should be in the radiative phase of pre-main sequence contraction. These stars have in addition infrared excesses. Basically two types of model have been invoked to explain the properties of Herbig Ae/Be stars; one involves the presence of an accretion disk and the other that of an active "chromosphere" plus wind, associated with a magnetic field.

From infrared photometry Strom et al. (1989) suggested that V 380 Ori belongs to the group of what they called "Class II" sources with a flux which decreased towards long wavelengths more slowly than that of a black body. These authors considered such sources to have a central object plus a disk. Hamann \& Persson (1992a) from the comparison of the CaII infrared triplet and the 
IR excess concluded that the line emitting envelopes are somehow related to the presence of disks.

A disk model with a central hole was supported by Hillenbrand et al. (1992) for most of the stars studied by them in order to better explain the infrared energy distribution. On the other hand Hartmann et al. (1993) show that this explanation raises serious problems of physical consistency, high accretion rates being necessary to produce the excess of emission. These authors suggest that, at least in some cases, it may rather be due to small grains of a dusty circumstellar nebula, transiently heated by stellar ultraviolet photons.

Böhm \& Catala (1995) explain their results on activity tracers with chromosphere plus wind models and give reasons for supposing that the presence of a disk is not necessary to sustain the observed activity.

The disk and/or the nebula need not be the only reason for the infrared excess; actually Leinert et al. (1997) find evidence for binarity for this and other Herbig Ae/Be stars from speckle interferometry. They also fit the infrared energy distribution with two stars plus infrared excess distributions.

In this uncertain situation, where it may be dangerous to generalize a given physical model to all stars belonging to the same "class", detailed studies of individual objects are required. It is in this context that we have studied V 380 Ori.

The star has a very rich emission line spectrum in the optical including, besides the Balmer lines, many metallic lines, of which those of Fe II are the most numerous followed by those of TiII. This star is associated with at least 3 Herbig-Haro objects, an extended $\mathrm{H}$ II region (NGC 1999), a bright crescent like reflection nebula around a dense globule, and sources of molecular emission (e.g. Shevchenko 1997). In this way it satisfies the conditions for belonging to the Herbig Ae/Be class. According to Böhm \& Catala (1995) its mass is $3.3 M_{\odot}$, and the effective temperature $9500 \mathrm{~K}$; they were unable however to find a rotation velocity due to the highly non-photospheric spectrum. Hillenbrand et al. (1992) derive slightly different parameters; the mass is $3.6 M_{\odot}$, while their effective temperature is $10700 \mathrm{~K}$, and corresponds to a spectral class of B9. In the frame of a model involving circumstellar accretion disk, in order to explain the strong infrared excess observed in the spectrum of V 380 Ori, Hillenbrand et al. (1992) determine a corresponding improbably high mass accretion rate of $510^{-6} M_{\odot} \mathrm{y}^{-1}$. We finally recall that V 380 Ori is also an X-ray source (see Strom et al. 1990, and references therein). In the radio a bipolar outflow is present with only red shifted components (e.g. Levreault 1988).

In spite of the many investigations, the nature of V 380 Ori and that of the Herbig AeBe stars in general, still is matter of controversy. In order to improve our knowledge on some aspects of this star, such as the strength and profile of the optical lines, the peculiar UV spectrum, and
Table 1. Observation log of V 380 Ori

\begin{tabular}{llllc}
\hline \multicolumn{1}{c}{ date } & telescope & instr. & $\begin{array}{c}\text { sp.range } \\
(\AA)\end{array}$ & $\begin{array}{c}\text { res }^{1} \\
\mathrm{~km} \mathrm{~s}^{-1}\end{array}$ \\
\hline 1978 Jul. 31 & IUE & lwr & $1200-1900$ & \\
1978 Oct. 28 & IUE & swp, lwr & $1200-3200$ & \\
1979 Apr. 17 & IUE & swp, lwr & $1200-3200$ & \\
1979 Sep. 26 & IUE & swp, lwr & $1200-3200$ & \\
1983 Dec. 15 & ESO 1.52 & IDS & $3920-5140$ & 64 \\
1983 Dec. 16 & ESO 1.52 & IDS & $4980-6220$ & 64 \\
1983 Dec. 17 & ESO 1.52 & IDS & $6060-7300$ & 65 \\
1983 Dec. 18 & ESO 1.52 & IDS & $7160-8390$ & 64 \\
1983 Dec. 19 & ESO 1.52 & IDS & $4000-8430$ & 460 \\
1983 Dec. 20 & ESO 1.52 & IDS & $5680-6910$ & 65 \\
1984 Apr. 16 & ESO 1.52 & IDS & $4200-8600$ & 460 \\
1984 Apr. 17 & ESO 1.52 & IDS & $4200-8600$ & 460 \\
1985 Mar. 16 & ESO 1.0 & InSb & JHKLM & \\
1985 Mar. 16 & ESO 1.0 & InSb & CVF & 70 \\
1985 Mar. 15 & ESO 1.52 & IDS & $4700-7065$ & 260 \\
1985 Mar. 16 & ESO 1.52 & IDS & $4000-5160$ & 64 \\
1985 Mar. 17 & ESO 1.52 & IDS & $4200-8800$ & 460 \\
1985 Apr. 8 & ESO 1.52 & IDS & $3950-5160$ & 64 \\
1985 Apr. 9 & ESO 1.52 & IDS & $4790-7200$ & 260 \\
1985 Apr. 10 & ESO 1.52 & IDS & $4790-8350$ & 300 \\
1989 Oct. 19 & IUE & swp, lwp & $1200-3200$ & \\
1995 Jan. 11 & OHP 1.52 & Aurelie & $4050-4490$ & 42 \\
1995 Jan. 12 & OHP 1.52 & Aurelie & $4890-5330$ & 36 \\
1995 Jan. 13 & OHP 1.52 & Aurelie & $5700-6575$ & 60 \\
1995 Jan. 15 & OHP 1.52 & Aurelie & $4450-4910$ & 39 \\
1995 Jan. 16 & OHP 1.52 & Aurelie & $5330-5770$ & 32 \\
\hline
\end{tabular}

Notes: ${ }^{1}$ Value at the central wavelength of the spectral range; ${ }^{2}$ The spectral resolution of CVF is given as $\lambda / \Delta \lambda$.

the star's activity and the wind tracers, we have collected new and archive observations of V 380 Ori which are here discussed. In particular, we obtained optical spectrograms with a resolution of $\sim 10^{4}$ which can also be a suitable basis for future more detailed studies of the process of formation of the heavy metal emission lines.

In Sects. 2 and 3 we present and discuss the observational data on V 380 Ori, which includes the long term spectroscopic monitoring at ESO and OHP, and the March 1985 coordinated program of simultaneous opticalinfrared spectroscopy and IR photometry at ESO. In addition, we analyse the archive IUE data, and study the energy distribution and the interstellar extinction. In Sect. 4 we discuss the main results of our work and compare with current models.

\section{Observations}

Table 1 summarizes the log of the observations used in this work. All but one observation are spectroscopic; a photometric infrared observation of March 1985 is included with the used filters indicated in Col. 4. For IDS and Aurelie spectra, the original resolving powers reported on the ESO and OHP user's manuals for the different configurations, 
were transformed in kilometers per second. IUE spectra were all obtained in low resolution mode.

Infrared data were obtained only in March 1985. For the photometric observations the integration time ranged from 1 to $2 \mathrm{~min}$, depending on the filter and on the selected signal to noise ratio. Narrow band spectrophotometric observations were performed on the same night with the Circular Variable Filter in the $1-5 \mu \mathrm{m}$ region. The integration time for each band was of $50 \mathrm{~s}$, and the full wavelength range was scanned in $82 \mathrm{~min}$. The $\mathrm{S} / \mathrm{N}$ ranges from 8 to 60 .

The optical spectrum of V 380 Ori was monitored at the $1.52 \mathrm{~m}$ ESO telescope with the B\&C spectrograph and IDS detector on several different epochs between December 1983 and April 1985. In each observing run several spectra were taken with different dispersions from 59 to $224 \AA \mathrm{mm}^{-1}$ in order to have both the details on the lines and the energy distribution for the whole spectral range of the instrument. The March 1985 spectroscopic observations were made simultaneously to the infrared observations. The observational data were calibrated using spectrophotometric standard stars. The April 1984 observations were obtained in bad weather conditions; the absolute flux calibration resulted of poor quality with different continuum levels from diffent exposures and different standard stars. The IDS spectra were reduced in Garching using the IHAP software package. In the ESO spectral images for the sky background correction we have taken the sky very close to the star so that also the emission from the nearby nebulosity has been subtracted.

More recently, in January 1995, we made new observations with the Aurélie spectrograph attached to the Coudé focus of the $1.52 \mathrm{~m}$ telescope of the Observatoire de Haute Provence, in order to provide spectrograms with a resolution high enough for the study of the line profile, and for an accurate line identification. During this run the observations were disturbed by very windy weather conditions which did not allow us to obtain reliable absolute fluxes. The problem of the calibration of these observations will be discussed in Sect. 3.2.

The S/N measured in wide "continuum" regions in all the optical spectra ranges from about 40 to more than 100 . But the actual $\mathrm{S} / \mathrm{N}$ is larger because the quoted values are to some extent affected by the presence of weak spectral lines.

Within this program, the ultraviolet spectrum of V 380 Ori was obtained with the International Ultraviolet Explorer $(I U E)$ at the ESA Villafranca Tracking Station on 1979 September 26 in the low resolution mode in both the short and long wavelength ranges. In addition, we have retrieved from the IUE archive all the other low resolution UV spectra taken in the period 1978-1989. All the IUE spetra used are listed in Table 1.

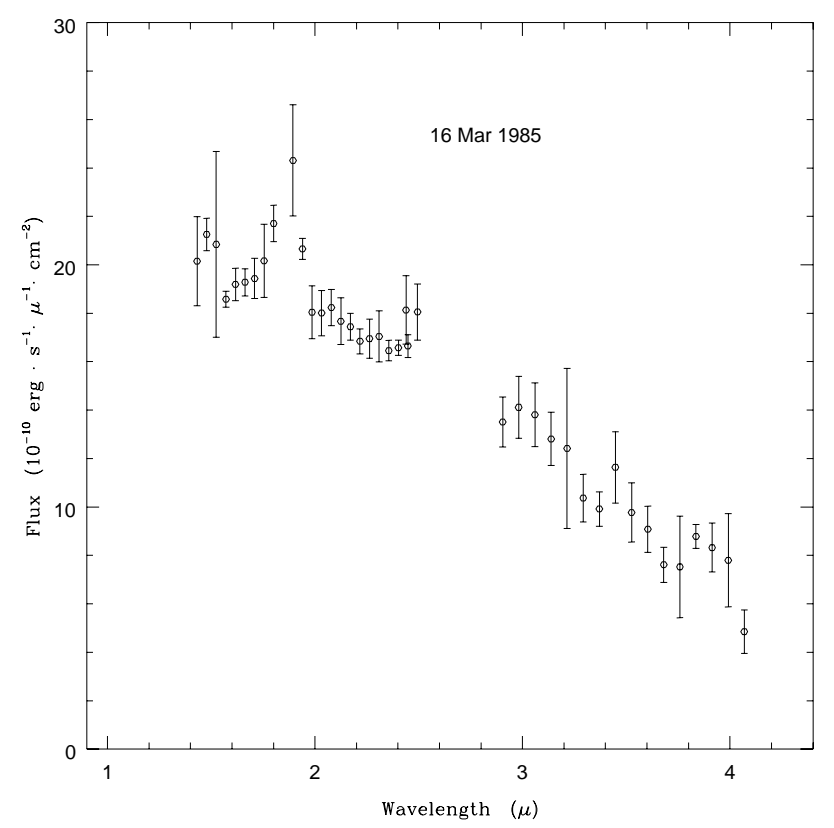

Fig. 1. The CVF IR observations of V 380 Ori in March 1985. Ordinates are fluxes in $10^{-10} \mathrm{erg} \mathrm{cm}^{-2} \mathrm{~s}^{-1} \mu^{-1}$

\section{Data analysis}

\subsection{Infrared spectrophotometry}

Simultaneous infrared and optical observations of V 380 Ori were secured in March 1985, so as to have its IR energy distribution at the same time as the optical one and to check for possible variability in the infrared magnitudes. The observed magnitudes and the $1 \sigma$ errors are reported in Table 2. Our photometric observations clearly show the presence of the large IR dust excess, which has been reported since the sixties by many authors. To check for a possible IR long term variability of V 380 Ori, we analysed the photometric broad band data available in literature. The data are summarized in Table 2 where, for very close observations we give the average values after checking that all the data fall well inside the observational errors. Though the comparison of observations taken with different instrumentation should be taken with care, it seems that long term oscillation (on a time scale of many months to years) might be present, while no systematic secular variations seem to be taking place.

During the March 1985 campaign, we have also secured the infrared spectrum of V 380 Ori which is shown in Fig. 1. Our spectrophotometric infrared data were obtained with a resolution higher than that of the other previous observations, mainly to search for emission and/or absorption features. But, like in the previous lower resolution observations, the spectrum appears featureless as if it were produced by thermal emission of dust grains (see Cohen 1980, and references therein). 
Table 2. Infrared photometry of V 380 Ori $^{*}$

\begin{tabular}{|c|c|c|c|c|c|c|c|}
\hline date & $J$ & $H$ & $K$ & $L$ & $M$ & $N$ & ref. \\
\hline 1965 Dec. 15 & $7.91(.04)$ & & $5.88(.03)$ & $4.38(.05)$ & & & 5 \\
\hline 1966 Oct. 8 & & $7.24(-)$ & $6.05(.03)$ & $4.76(.05)$ & & & 5 \\
\hline 1966 Nov. 2 & $8.24(.04)$ & $7.49(-)$ & $6.12(.03)$ & $4.71(.05)$ & & & 5 \\
\hline 1981 & $8.0 \quad(.1)$ & $7.0 \quad(.1)$ & $5.9 \quad(.1)$ & $4.2 \quad(.1)$ & $3.7 \quad(.1)$ & & 1 \\
\hline 1981 Dec. & $8.16(.03)$ & $7.13(.03)$ & $6.11(.03)$ & $4.42(.05)$ & & & 6 \\
\hline 1982 Jan. & $8.17(.03)$ & $7.12(.03)$ & $6.08(.03)$ & $4.75(.05)$ & & & 6 \\
\hline 1982 Nov. & $8.13(.03)$ & $7.08(.03)$ & $6.05(.03)$ & $4.62(.05)$ & & & 6 \\
\hline 1983 Mar. & $7.98(.03)$ & $6.90(.03)$ & $5.84(.03)$ & $4.53(.05)$ & & & 6 \\
\hline 1984 Jan. & & $7.18(.2)$ & & & $4.07(.2)$ & $2.22(.1)$ & 4 \\
\hline 1985 Mar. 16 & $8.10(.02)$ & $7.01(.03)$ & $5.98(.03)$ & $4.50(.06)$ & $4.05(.10)$ & & 2 \\
\hline 1987 Sep. & $8.09(.03)$ & $6.98(.03)$ & $5.96(.03)$ & $4.56(.05)$ & $3.80(.15)$ & $1.77(.15)$ & 3 \\
\hline
\end{tabular}

Notes to the table: ${ }^{*}$ In parentheses the quoted errors. Sources: ${ }^{1}$ Lorenzetti et al. (1983); ${ }^{2}$ Present work; ${ }^{3}$ Hutchinson et al. (1994); three observations for JHKL; ${ }^{4}$ Davies et al. (1990); ${ }^{5}$ Mendoza (1968); ${ }^{6}$ Kilkenny et al. (1985).

\subsection{The optical spectrum}

The overall optical spectrum of V 380 Ori appears substantially constant during the period covered by our observations, except for some cases discussed below. In particular the equivalent widths of the emission lines of both ESO and OHP spectra are comparable with each others and with those of November 1991 reported by Shevchenko (1997). We have also found that the continuum flux and slope did not change during 1983-1985. In Fig. 2 we present some examples of the spectra in different spectral regions. In each panel only spectrograms with the same resolution are compared. In the upper panel the April 1984 appears weaker than expected being of lower quality, as explained in Sect. 2.

Concerning the January 1995 OHP spectra, as discussed above, we could not perform the absolute calibration. Nevertheless, after the standard reduction procedure, the continuum slope of each OHP spectrogram well agrees with that of the ESO spectrograms. Taking into account the stability of our spectroscopic data and after verification that no major photometric variation occurred in the last decade, we corrected the continuum level of the OHP spectra by constant factors in order to have them overlapped onto the ESO spectra, with a mean continuum level of around $210^{-13} \mathrm{erg} \mathrm{cm}^{-2} \mathrm{~s}^{-1} \AA^{-1}$, being confident that this procedure does not produce significant systematic errors on the true values of the absolute fluxes of the lines. In fact, we intend to use in a forecoming paper the higher resolution OHP spectra for the analysis of the flux of metallic emission lines using the statistical method of the Self Absorption Curve (Friedjung \& Muratorio 1987), in order to determine the physical conditions of the line emitting region.

Figure 3 shows the 1995 optical spectrum of V 380 Ori which appears very rich in emission lines, mainly due to metals in low ionization stages, and a few absorption lines of photospheric (such as the Stark-broadened Balmer lines and weak Fe I and Si II lines), or interstellar origin (e.g. the Na I yellow doublet, the DIB at $6275 \AA$ ). Most emission lines appear broad with a mean FWHM of about $150 \mathrm{~km} \mathrm{~s}^{-1}$, which in some cases causes serious problems of line blending.

Previous spectroscopic studies of V 380 Ori were mostly limited to the description of some particular lines, or have presented mean values of radial velocities and of line widths. Line lists have also been published (see Shevchenko 1994 and references therein), but in these identifications normally only the line considered to be the main contributor is reported. Since in V 380 Ori the lines are intrinsically broad, close blends cannot be easily separated, but secondary contributors may appear as bumps on the wings of the observed emissions in sufficiently high resolution spectra. In fact, most of the emission lines in our higher resolution OHP spectra present asymmetries that can be explained either by the contribution of the same line from different emitting regions with different radial velocities, or by another element.

For the purposes of our work on metallic emission lines we felt the need of making a line list as accurate as possible, and have therefore made a new line identification using a semiautomatic iterative procedure, based on Kurucz (1994) line list, which was run in the MIDAS environment. For each emission feature we measured the central wavelength and the flux above the nearby continuum by means of a Gaussian fit, and made a first identification of the possible contributors by an automatic selection from the list. Of the lines in the vicinity of the observed spectral feature we accepted only those belonging to transitions of ionized metals, and of a few neutral species, whose excitation potential and line strength are such that the lines are expected to be detectable. The few well known lines of $\mathrm{H}$ I, He I, Na I, CaI, and Mg II were excluded from this automatic search. We have measured the flux, FWHM and position of the expected contributors to emission blends with multiple Gaussian fits. It turned out that many asymmetric lines, which were attributed to a single line in previous works on V 380 Ori based on smaller resolution or lower 

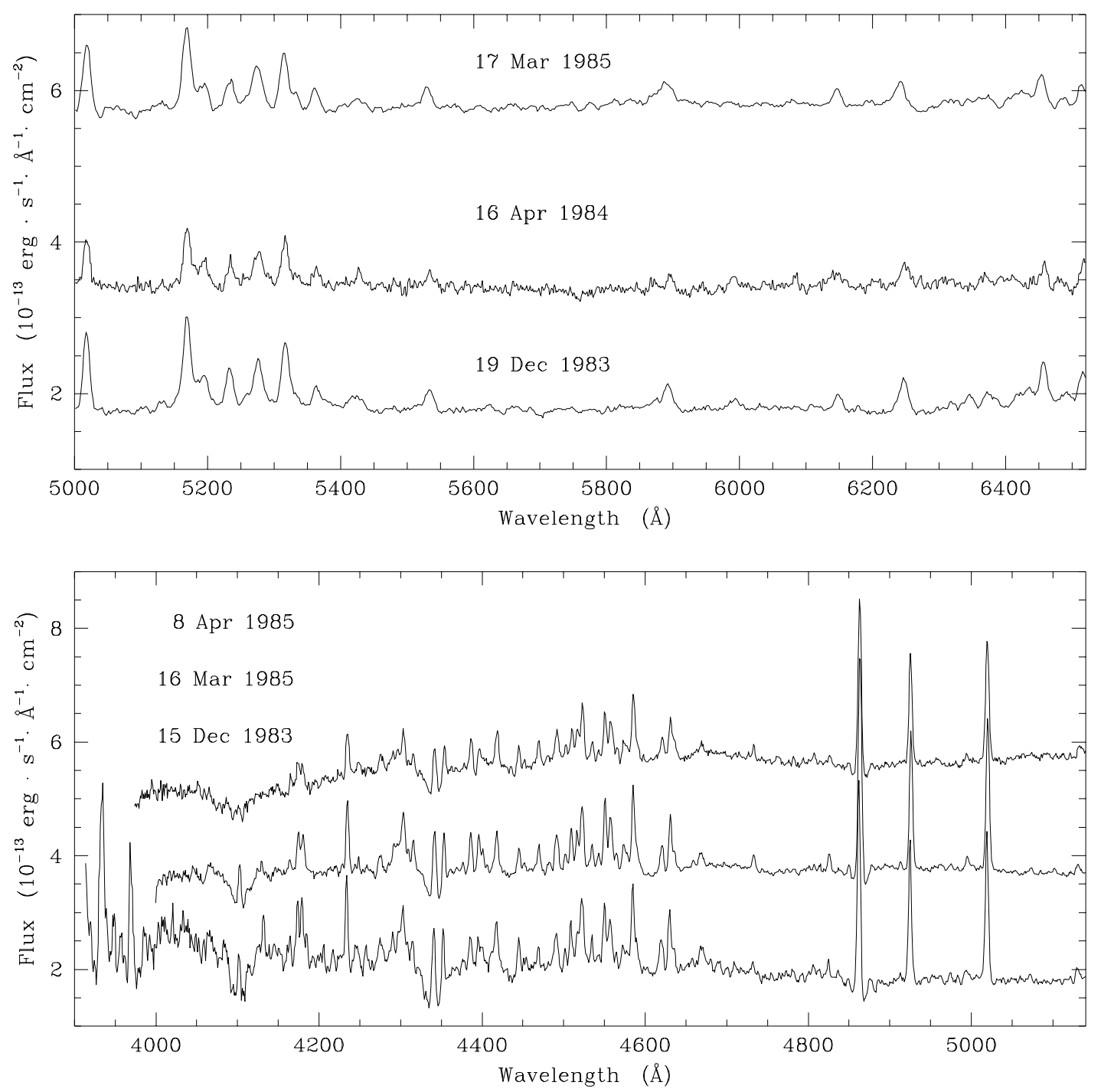

Fig. 2. Comparison of selected spectral regions of V 380 Ori observed at ESO during 1983-1985. Fluxes are in $10^{-13} \mathrm{erg}^{-2}$ $\mathrm{s}^{-1} \AA^{-1}$, not corrected for the interstellar extinction. The upper tracings are vertically shifted by steps of $210^{-13} \mathrm{erg} \mathrm{cm}^{-2} \mathrm{~s}^{-1}$ $\AA^{-1}$. The April 1984 spectrum is of poor photometric quality (see text)

$\mathrm{S} / \mathrm{N}$ spectra, are in most cases well fitted by blends of two or more lines. No correction was made for the possible presence of photospheric absorption lines below the emission observed features, except for the broad $\mathrm{H}$ absorptions. The final selection was made in the usual way by checking the multiplet tables and by comparing with previously published identifications.

Table 3 gives the emission line spectrum of V 380 Ori in January 1995, which includes emission lines of H I, He I, [O I], Na I, Mg II, Si II, CaI, VII, Ti II, Cr II, [FeII], Fe II, and Ni II. In Table 3 we give for the line blends the wavelength and flux (not reddening corrected) of each contributor to the blend. We estimate an error of less than $10 \%$ for the strongest lines, $10 \%$ to $40 \%$ for the weakest lines.
We remember that Table 3 is available at CDS (see footnote to the abstract).

Most of the emission lines belong to Fe II permitted transitions with heliocentric radial velocity of about $+37 \mathrm{~km} \mathrm{~s}^{-1}$; the profile of the strongest lines is characterized by a hump on the blue side of the central symmetric emission peak (see Figs. 4a-c), which we were unable to otherwise identify. The line shape suggests the presence of two separate components, rather than a line broadening due for instance to a continuous velocity gradient. In the weaker lines the blue component is confused with the principal emission and produces a blue asymmetry. In order to take this into account, we have fitted each line to two components with the nearly same width $(F W H M \simeq$ 

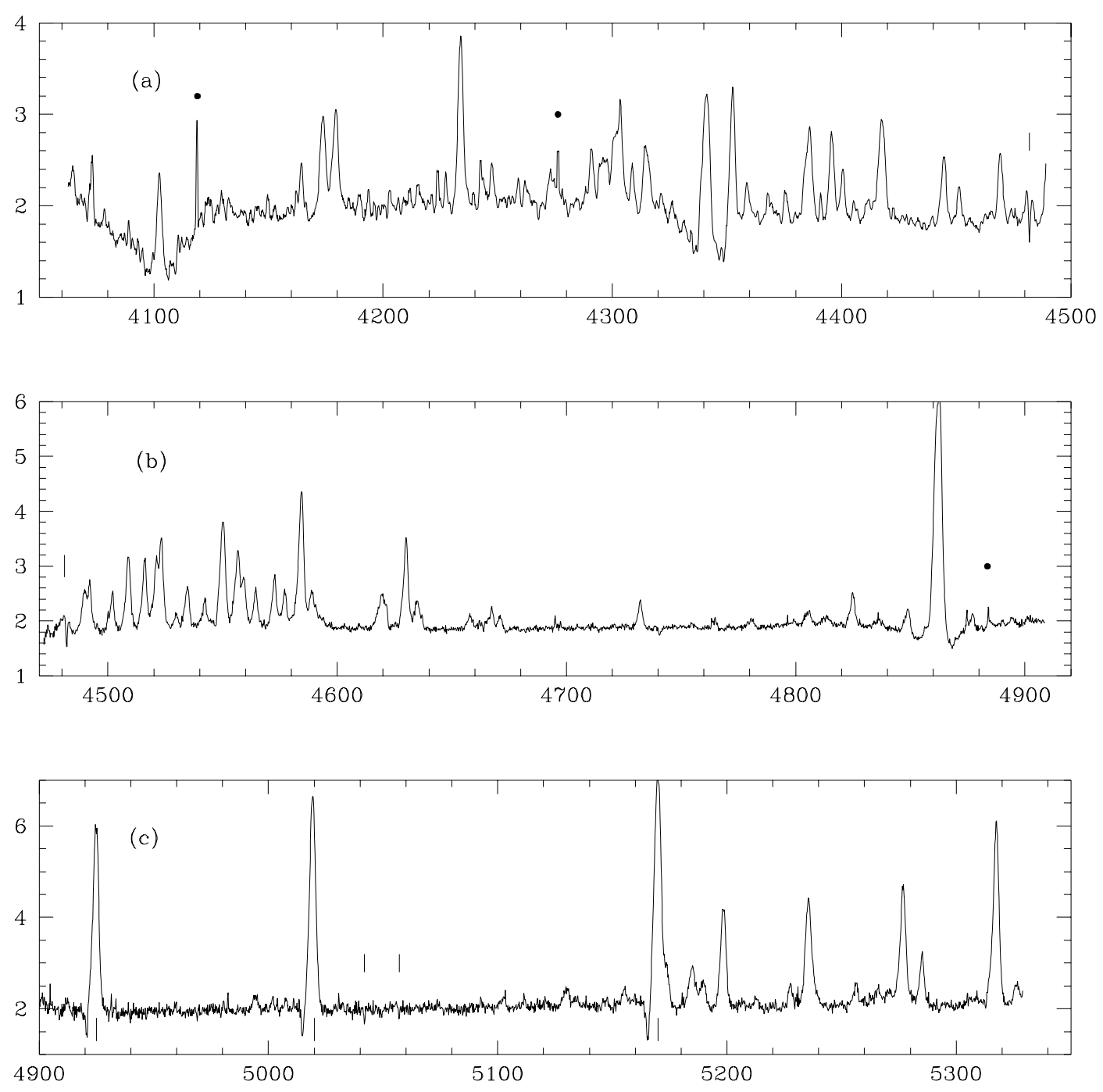

Fig. 3. The optical spectrum of V 380 Ori observed in January 1995. The scales are the same as in Fig. 2. The fluxes are normalized as explained in Sect. 3.2. In the tracings dots indicate spikes. In Figs. 3a and 3b the vertical bars correspond to Mg II $4481 \AA$ line. In Fig. 3c the PCygni profiles of Fe II (42) and the two photospheric absorptions of Si II (5) are indicated

$150 \mathrm{~km} \mathrm{~s}^{-1}$ ), a principal one centred at the stellar velocity, and a blue component. From the fit we found for the latter one a nearly constant blueshift of $-140 \mathrm{~km} \mathrm{~s}^{-1}$ with respect to the principal emission (Figs. 4a-c). The strong Fe II multiplet 42 lines have P Cygni absorption components centred at $-250 \mathrm{~km} \mathrm{~s}^{-1}$ with respect to the emission peak, and extending to about $-300 \mathrm{~km} \mathrm{~s}^{-1}$ (Fig. 3). This signature of outflow is an indication of line formation in a cool wind. Since no P Cygni absorption is seen in the Fe II lines other than multiplet 42 , the wind should be rather teneous. On the other hand, the relative strength of the Fe II principal emission components within multiplets, and the preliminary results obtained from the analysis with the SAC method on the whole set of multiplets
(Muratorio et al. 1998) clearly indicate that they originate in an optically thick medium, which, if were placed in front of the stellar photosphere, would produce a strong line absorption. Therefore, the absence of $\mathrm{P}$ Cygni absorption in the other Fe II lines, combined with the emission line broadness, is strongly suggestive of line formation in a geometrically bounded rotating region such as an equatorial disk, seen at a rather large inclination angle, rather than in a wind.

In addition to the permitted transitions, we have identified several weak narrow Fe II forbidden lines $\left(F W H M \sim 70-100 \mathrm{~km} \mathrm{~s}^{-1}, v_{\text {hel }} \sim 35 \mathrm{~km} \mathrm{~s}^{-1}\right)$, that might be expected to be formed in the outer less dense parts of the $\mathrm{Fe}^{+}$emitting region. Their narrowness 

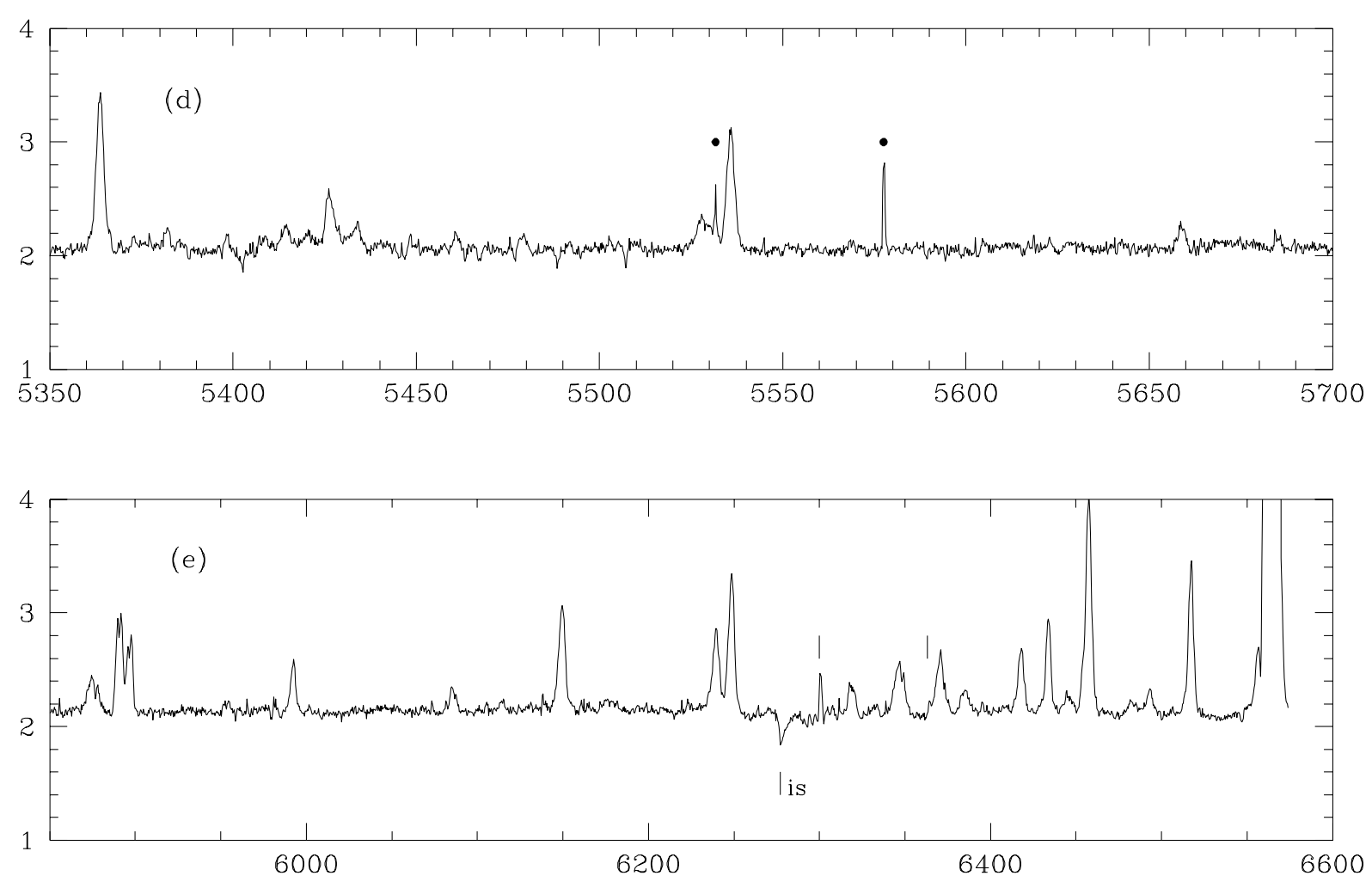

Fig. 3. continued. The optical spectrum of V 380 Ori observed in January 1995. In Fig. 3e the bars put in evidence the [O I] lines

indicates line formation in a region different from that where the Fe II multiplet $42 \mathrm{P}$ Cygni absorptions are formed. They are most probably formed in the less dense outer parts of the disk where the Keplerian velocity is much smaller.

[O I] is present on the OHP spectrum with a narrow $6300 \AA$ line $\left(F W H M \sim 84 \mathrm{~km} \mathrm{~s}^{-1}\right.$, not corrected for the instrumental profile); the equivalent width is $W_{\mathrm{eq}}^{\mathrm{e}} \sim 0.4 \AA$; no asymmetry is detectable. Particular care was taken in verifying the goodness of the wavelength scale. We obtained for this line a heliocentric radial velocity of $+22 \pm 4 \mathrm{~km} \mathrm{~s}^{-1}$, the same as the interstellar Na I D lines (see below), which is indicative of a forming region at systemic velocity as also suggested by Böhm \& Catala (1994) for other Ae/Be systems.

The $6363 \AA$ component is clearly detectable on the blue wing of the Fe II $6369 \AA$ emission line (Fig. 3). The 6300 line is also visible on the 1983 spectrum. Similar width and strength of the $6300 \AA$ emission were found by Hamann (1994). The line was not detected in January 1992 by Böhm \& Catala (1994), while Corcoran \& Ray (1998) found the line to be much stronger $\left(W_{\mathrm{eq}}^{\mathrm{e}}=0.97 \AA\right)$ in a spectrum taken only one month before our OHP observations. The intrinsic weakness of the $[\mathrm{O}$ I $]$ lines, the variety of the instruments used by different groups and the lacking of systematic observations makes it difficult either to confirm a possible short time variability, or to give a realistic interpretation about the origin of this line.
The hydrogen Balmer series is present on our spectra from $\mathrm{H} \alpha$ to $\mathrm{H} \delta$. For $\mathrm{H} \alpha$ a $F W H M=260 \mathrm{~km} \mathrm{~s}^{-1}$ was measured on the OHP and ESO spectra of 17 and 20 December 1983. Starting from $\mathrm{H} \beta$ the emission emerges from the photospheric absorption. In spectra with sufficiently high resolution we measured the equivalent widths with respect to the base of the lines. In the low resolution spectra the equivalent width includes the absorption; in order to make a better comparison with the others we degraded the spectra of higher resolution and measured again the equivalent widths. For each run the differences between the values from original low resolution spectra and those from "degraded" spectra resulted lower than the uncertainties. The measured equivalent widths of the Balmer lines are presented in Table 4. In the OHP spectra all the emissions but $\mathrm{H} \alpha$ appear asymmetric with a red peak and a bump on the blue wing. The heliocentrinc velocities from $\mathrm{H} \alpha$ to $\mathrm{H} \delta$ at the FWHM are $+39,+42,+32$ and $+37 \mathrm{~km} \mathrm{~s}^{-1} \pm 5 \mathrm{~km} \mathrm{~s}^{-1}$, respectively, close to that of permitted Fe II. The peaks are redshifted by about $25 \mathrm{~km} \mathrm{~s}^{-1}$. The observed differences can only partly be attributed to the different observing conditions, and are clearly indicative of a significant variablity, as also confirmed by the analysis of published data, which for $\mathrm{H} \alpha$ range from an equivalent width of $58 \AA$ in November 1981 (Finkenzeller \& Mundt 1984), to about $80 \AA$ in January 1992 (Böhm \& Catala 1995). A value of $107 \AA$ was measured by Garrison 

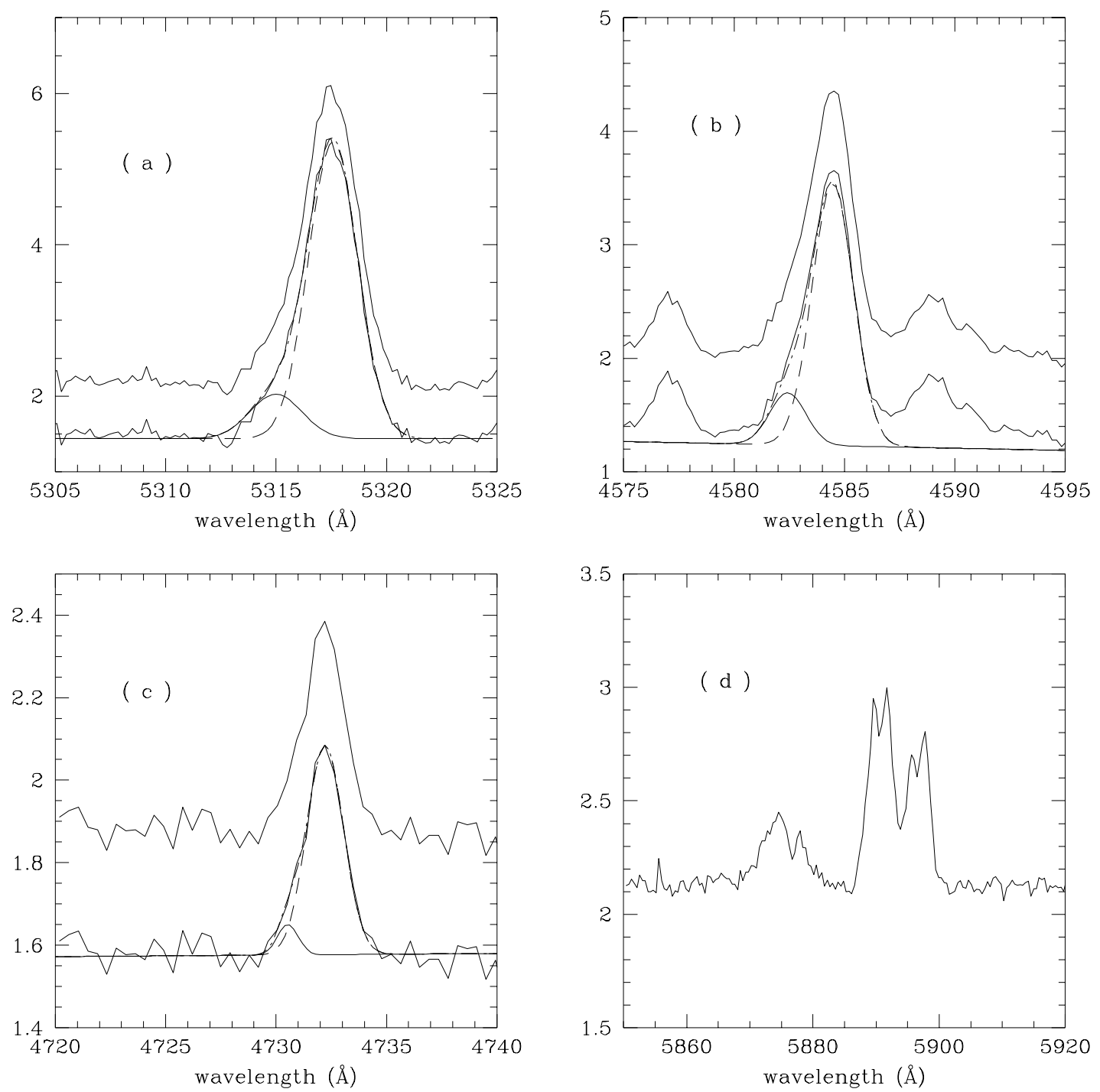

Fig. 4. Some spectral features in the January 1995 spectrum of V 380 Ori: a-c) Fe II emission lines fitted with two Gaussians. For a better evaluation of the fit the observed spectrum is also reproduced vertically shifted. d) Spectral region of the He I $5876 \AA$ line and the NaI resonance doublet. The scales are the same as Fig. 2

\& Anderson (1977) on plate spectra. Again nothing can be so far said about the time scale of the variations.

Helium is present in all our ESO and OHP spectra with the emission lines at $5876 \AA$ and $6678 \AA$. No other He I emission lines such as 4471 and $4713 \AA$ have been found in the OHP spectra. The $4713 \AA$ line seems to be weakly present in the 1983 ESO spectrum. The $4921 \AA$ and $5015 \AA$ lines, if present, are masked by the very strong Fe II lines of multiplet 42 .

The higher resolution OHP spectrum show the $5876 \AA$ line as a wide asymmetric emission $(F W H M \sim$ $380 \mathrm{kms}$, Fig. 4d), the mean of the wavelengths at half maximum intensity gives a heliocentric radial velocity of about $-22 \mathrm{~km} \mathrm{~s}^{-1}$. A sharp absorption is clearly visible at $+48 \mathrm{~km} \mathrm{~s}^{-1}$ on the red wing of the line. The flux of the $5876 \AA$ emission line corresponds to an equivalent width of $1.08 \AA$, a value which is comparable with that measured in our ESO observations of December $1983\left(W_{\mathrm{eq}}^{\mathrm{e}}=\right.$ $1.00 \AA$ ), and of April $1985\left(W_{\mathrm{eq}}^{\mathrm{e}}=1.26 \AA\right)$, while the line appeared weaker $\left(W_{\mathrm{eq}}^{\mathrm{e}} \sim 0.75 \AA\right.$ ) in the spectrum of 15 March 1985. This He I line was also in emission in 1991 (Shevchenko 1994), while it was clearly in absorption in 1981 (Finkenzeller \& Mundt 1984). Neither absorption nor emission was found by Böhm \& Catala (1995) at $5876 \AA$ A on 16 January 1992. Our ESO observations show that also the He I $6678 \AA$ line is in emission with $W_{\mathrm{eq}}^{\mathrm{e}} \sim 0.5-0.7 \AA$. Corcoran \& Ray (1995) report that in February 1990 this line was present as a broad emission $(F W H M=$ 
Table 4. Equivalent widths $(\AA)$ of the Balmer lines

\begin{tabular}{ccccc}
\hline date & $\begin{array}{c}\mathrm{H} \alpha \\
\pm 5\end{array}$ & $\begin{array}{c}\mathrm{H} \beta \\
\pm 1\end{array}$ & $\begin{array}{c}\mathrm{H} \gamma \\
\pm 0.7\end{array}$ & $\begin{array}{c}\mathrm{H} \delta \\
\pm 0.5\end{array}$ \\
\hline Dec. 1983 & 90 & $13.5-7$ & 5.0 & 2.6 \\
Apr. 1984 & 82 & 6.8 & $>1.5$ & \\
Mar. 1985 & 84 & $14.7-8.8$ & 4.9 & 3.0 \\
Apr. 1985 & 78 & $11.0-6.5$ & 3.5 & 2.4 \\
Jan. 1995 & $>78$ & $13.2-8.0$ & 5.2 & 2.5 \\
\hline
\end{tabular}

Notes: The second value of $\mathrm{H} \beta$ was obtained from low resolution and degraded spectra (see text).

$340 \mathrm{~km} \mathrm{~s}^{-1}$ ) at heliocentric velocity of $+40 \mathrm{~km} \mathrm{~s}^{-1}$, splitted into two nearly symmetric components by a central absorption, which again confirms the large variability in both strength and shape of the He I lines in V 380 Ori.

The Mg II $\lambda 4481$ emission which is present in all our observations, in the higher resolution OHP spectra has nearly the same profile of He I $5876 \AA$ with a broad emission split asymmetrically by a central absorption (Fig. 3) at heliocentric radial velocity close to that of the He I central absorption.

The Na I resonance doublet is also present with two broad emissions which are both split by a narrow absorption at $+24 \mathrm{~km} \mathrm{~s}^{-1}$ which is interstellar in origin (Fig. 4d).

In the ESO spectrum the CaII $h$ and $k$ violet doublet lines have very strong emission components (Fig. 2), while the near-infrared is characterized by the emission lines of the Paschen series, Mg II $\lambda 7896$, the O I $7771 \AA$ and $8446 \AA$ lines, and the very strong Ca II m.2 triplet. A comparison with previous observations (Herbig \& Soderblom 1980, data obtained in 1970 and 1978; Shevchenko 1994, data obtained in 1991; Böhm \& Catala (1995), data obtained in 1992) indicates quite constant equivalent widths of $\mathrm{OI}$ and $\mathrm{Mg}$ II lines and a slight variability of the Ca II triplet. The intensity ratio of the calcium triplet lines, corrected for the contribution of the Paschen lines, is close to unity in agreement with the other observations, and suggests line formation in an optically thick medium.

\subsection{The ultraviolet spectrum}

The ultraviolet spectrum of V 380 Ori was observed with $I U E$ at many epochs within different programmes: 1978 July 31 and October 28, 1979 April 17 and September 26, and 1989 October 19. The ultraviolet spectrum of V 380 Ori is characterized by the presence of many absorption features, and by a sharp flux decrease shortwards of about $1300 \AA$ (see Fig. 5). In spite of the rich emission line spectrum observed in the optical, the Mg II $2800 \AA$ doublet seems to be the only emission feature visible at low resolution, while Fe II is present with strong absorption features. The line identification of the UV spectrum of V 380 Ori includes absorption lines of O I, C I, C II, C IV, Si II, Si III, Si IV, Al II, AlıII, and especially Fe II. C IV and Si IV are probably only of interstellar origin. The most prominent features are those of Fe II with a deep and broad absorption at $2750 \AA$ and the flux cutoffs at 2410 and $2630 \AA$ due to the blends of many resonance and low excitation lines. The low resolution does not allow us to determine whether the absorption features are also produced by the same expanding material which produces the $\mathrm{P}$ Cygni absorptions of the optical Fe II multiplet 42 lines, as discussed above. Hence the spectral features may not be good indicators for spectrum and luminosity classification. To overcome this problem, we have compared the UV spectrum of V 380 Ori with that of standard stars from the IUE Low-Dispersion Spectra Reference Atlas (Heck et al. 1984). We have found that the short wavelength cut off near $1300 \AA$ is typical of B9-A0 stars (where it is also due to the strong red wing of Ly $\alpha$ ). The absorption features, and most notably those of Fe II, are more typical of a later spectral type, and are at any rate much deeper than in main sequence stars.

We are therefore brought to the conclusion that the ultraviolet absorption spectrum is formed in an envelope, cooler than the underlying stellar photosphere, whose partly overlapping lines hide the emission components.

\subsection{Interstellar extinction and energy distribution}

A depression near $2175 \AA$ due to the interstellar band is clearly recognisable in all the UV spectra of V 380 Ori, partly affected on both wavelength sides by the shell absorptions (Fig. 5). Using the mean galactic extinction law, we have found that the feature disappears for $E_{B-V}=$ $0.20 \pm 0.05$. This value is however in disagreement with the colour excess which can be derived from the optical energy distribution. In fact the literature generally gives a colour index of $B-V \approx+0.5$ for the star (e.g. Finkenzeller \& Mundt 1984; Hillenbrand et al. 1992), which, if associated with an effective temperature of $\sim 10000 \mathrm{~K}$, would imply a colour excess of $E_{B-V} \sim 0.5$. This is also in agreement with the strength of the DIB at $6280 \AA$ observed in the OHP spectrum. No multicolour photometry has been made simultaneously to the $I U E$ and optical spectroscopic observations.

It should be recalled that the broad band photometry does not directly reproduce the continuum because of the contribution of the many emission lines. Using our spectrograms we have found that the emission lines contribute by nearly the same amount, $\sim 10 \%$, to both $B$ and $V$, so that the colour excess is not significantly altered by the emission lines. We think that the difference between the ultraviolet and visual colour excesses must be attributed to an anomalous interstellar extinction law in the $\mathrm{V} 380$ Ori region. This is not an unexpected result since anomalous extinction curves have been in many cases found in regions of star formation.

We have analysed the depth of the $2175 \AA$ band with a code kindly provided us by A. Cassatella, which adopts the 


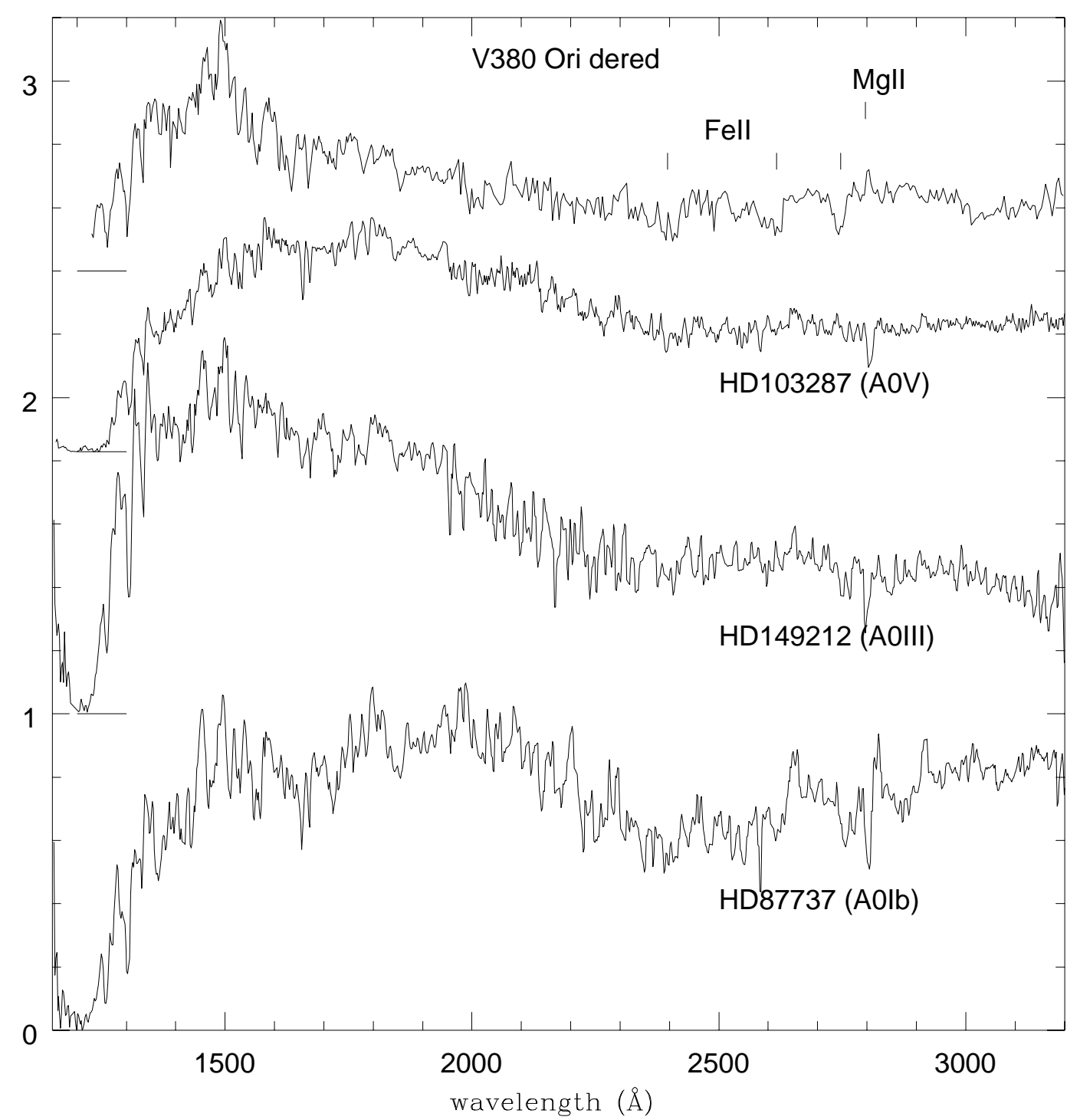

Fig. 5. Comparison of the UV low resolution spectrum of V 380 Ori with that of spectroscopic standard stars. The offset spectra are, from top to bottom: the dereddened spectrum of V 380 Ori observed with IUE in September 1979, and the IUE spectra of A0V, A0III and A0I standard stars. The spectrum of V 380 Ori is dereddened assuming $E_{B-V}=0.5$ and a $2175 \AA$ interstellar band 2.5 times weaker than for the mean Galactic extinction law. Ordinates are fluxes in $10^{-14} \mathrm{erg} \mathrm{cm}^{-2} \mathrm{~s}^{-1} \AA^{-1}$

parametrization proposed by Fitzpatrick \& Massa (1986). We found satisfactory results assuming a colour excess of $E_{B-V}=0.5$ with a $2175 \AA$ band 2.5 times weaker than the galactic one.

\section{Discussion}

The long term spectroscopic monitoring presented in this work and the comparison with the previous ones, show that V 380 Ori is a very active star. The many and somehow contrasting peculiarities of this star can be explained in the framework of a model of a central star plus accretion disk, as could be suggested in particular by the anomalous energy distribution. There are also evidences of the presence of outflowing matter from the star and/or from the disk.

The presence of an extended emitting region is supported by the many broad metallic permitted lines that crowd the optical spectral region. In particular, the width of the principal components of Fe II combined with their large optical thickness and with the absence of $\mathrm{P}$ Cygni absorptions in all lines but those of multiplet 42 , is interpreted as due to Keplerian motion in an accretion disk. Assuming for the central star a mass of $3.3 M_{\odot}$ (Böhm \& Catala 1995), a value of $\sim 110^{13} \sin ^{2} i \mathrm{~cm}$ is found for the mean size of the Fe II emitting region. A teneous high 
velocity wind is most probably causing the few Fe II $\mathrm{P}$ Cygni absorptions. The broad blue shifted emission components of FeII seem to be produced in a region less extended than that of the principal components. Their blueshift is about half that of the P Cygni absorption components seen for the lines of multiplet 42 of Fe II. It seems reasonable to suppose formation of the blue components in the inner regions of the wind. In this case we would expect that the emission from the receding matter is occulted by the disk, in agreement with the accretion wind disk model proposed by Corcoran \& Ray (1998).

A puzzling problem is represented by the ultraviolet spectral region where, neither the line spectrum, nor the interstellar extinction seem to agree with the optical data. The i.s. extinction law results to be anomalous and the only possible consistency with the optical $E_{B-V}$ value is obtained assuming that the strength of the $2175 \AA$ band is much weaker than the galactic one. The line spectrum better fits a spectral type later than B9, and appears to be most probably produced by absorption in outer regions. Once the possible effect of the overlapping of absorption and emission components of metallic lines is taken into account, the UV continuum energy distribution appears to be in agreement with the stellar effective temperature.

The stellar activity is marked by the presence of the $\mathrm{O}$ I and Ca II emissions, and by the variability especially of the hydrogen and helium lines.

An intriguing feature is the He I $\lambda 5876 \AA$ line, for which we always detected an emission component, while in other epochs the line was either only in absorption or not present at all. We verified also on literature spectra that the helium absorpion lines, when visible, always presented the same heliocentric radial velocity $\left(\sim 45 \mathrm{~km} \mathrm{~s}^{-1}\right)$; this does not necessarily imply for the star a surface temperature higher than that estimated from the photospheric absorptions profiles of Balmer lines $(\sim 10000 \mathrm{~K})$. Given the presence of an emission component, a chromospheric origin would be a better explanation, as suggested by Böhm \& Catala (1995) for other Herbig Ae/Be stars. Another possibility is the presence of a stationary hot circumstellar region.

Different solutions are possible to explain the emission component, variable both in intensity and in radial velocity, as for example the presence of an unstable hot region (hot spot?) close to the stellar surface, produced by irregular accretion processes. Alternatively, the He I emission might be produced by hot wind ejected mainly from the polar regions of the star or from the inner parts of a circumstellar disk in Keplerian rotation around the star (see also Böhm \& Catala 1995). The width of the emissions (larger than the hydrogen lines) seems to favour the latter hypothesis. Hence the helium lines forming region is most probably located close to the star at the base of an accelerated wind which cools at larger distances where the Fe II blue components should be formed.
It would be important to give some more support to this possibility, looking for the variation of the blue Fe II emission component and by searching any correlation with the He I lines. We also think that the precise localization of the formation region and the time scales of the variability of the helium lines in the Herbig $\mathrm{Ae} / \mathrm{Be}$ is an important problem in the framework of modelling the external layers, which would require high quality and systematic observations for all the stars of this category.

Acknowledgements. This research has made use of the Simbad database, operated at CDS, Strasbourg, France and $f$ the archives of the Astronomical Data Center of the NASA Goddard Space Flight Center. We are grateful to Angelo Cassatella for discussions on the interstellar extinction law, We also thank the referee T. Böhm for a careful reading the manuscript and helpful suggestions, and to Herman Tijn A Djie for stimulating discussions. M. Friedjung, G. Muratorio, C. Rossi and R. Viotti were partially supported by the 19961997 CNR-CNRS exchange programme.

\section{References}

Böhm T., Catala C., 1994, A\&A 290, 167

Böhm T., Catala C., 1995, A\&A 301, 155

Cohen M., 1980, MNRAS 191, 499

Corcoran D., Ray T.P., 1995, A\&A 301, 729

Corcoran M., Ray T.P., 1998, A\&A 331, 147

Davies J.K., Evans A., Bode M.F., Whittet D.C., 1990, MNRAS 347,517

Finkenzeller U., Mundt R., 1984, A\&AS 55, 109

Fitzpatrick E.L., Massa D., 1986, ApJ 307, 286

Friedjung M., Muratorio G., 1987, A\&A 188, 100

Garrison L.M, Anderson C.M., 1977, ApJ 218, 438

Hamann F., 1994, ApJS 93, 485

Hamann F., Persson S.E., 1992a, ApJ 394, 628

Hamann F., Persson S.E., 1992b, ApJS 82, 285

Hartmann L., Kenyon S.J., Calvet N., 1993, ApJ 407, 219

Heck A., Egret D., Jaschek M., Jaschek C., 1984, IUE LowDispersion Spectra Reference Atlas, ESA SP-1052

Herbig G.H., 1960, ApJS 4, 337

Herbig G.H., Soderblom D.R., 1980, ApJ 242, 628

Hillenbrand L.A., Strom S.E., Vrba F.J., Keene J., 1992, ApJ 397,613

Hutchinson M.G., Albinson J.S., Barret P., Davis J.K., Evans A., Goldsmith M., Maddison R.C., 1994, A\&A 285, 883

Kilkenny D., Whittet D.C., Davies J.K., Evans A., Bode M.F., Robson E.I., 1985, South Afr. Astr. Obs., Circ. 9, 55

Kurucz R.L., 1994 (private communication)

Leinert C., Richichi A., Haas M., 1997, A\&A 318, 472

Levrault, 1988, APJS 67, 283

Lorenzetti D., Saraceno P., Strafella F., 1983, ApJ 264, 554

Mendoza E., 1968, ApJ 151, 977

Muratorio G., Rossi C., Friedjung M., Viotti R., 1998, in: B[e] Stars, Hubert A.M. \& Jaschek C. (eds.). Kluwer, Dordrecht (in press)

Shevchenko V.S., 1994, Astron. Rep. 38, No. 4, 505 transl. from Astronomicheskii Zhurnal 71, No. 4, 572

Strom K.M., Newton G., Strom S.E., et al., 1989, ApJS 71, 183

Strom K.M., Strom S.E., Wilkin F.P., et al., 1990, ApJ 362, 168 